\title{
A Simulated Annealing Approach to Evaluate Long Term Marginal Costs and Investment Decisions
}

\author{
Maria Teresa Ponce de Leão \\ mleao@inescn.pt \\ Engineering Faculty of University of Porto \\ INESC Porto - Inst. de Engenharia de Sistemas e Computadores \\ Largo de Mompilher, no. 22, 4050-392 Porto, Portugal \\ Phone: +351.2.2..2094230 Fax: +351.2.2.2084172
}

\begin{abstract}
In this paper we describe a multiobjective formulation for the long term planning of distribution networks considering a number of important features. The model admits fuzzy representations for loads and evaluates nodal long term marginal prices. It integrates a number of criteria related to investment, operational and reliability costs, risk index measuring the ability to accommodate load uncertainties and the remuneration collected using long term marginal prices. After using a Simulated Annealing approach to identify efficient expansion plans, it is finally conducted a decision analysis in order to select the most adequate plan. At a final section, we illustrate the formulation with a case study based on a Portuguese distribution network.
\end{abstract}

Keywords: Marginal Prices, Simulated Annealing, Electricity Markets, Regulatory Policies.

\section{INTRODUCTION}

Re-regulation of electricity business still presents great challenges namely on issues related to the collection of the remuneration of transmission and distribution wiring companies. The relevance of this topic comes from the decoupling of activities that were traditionally integrated in the utilities. In this case, the ownership, operation, expansion and maintenance of networks are assigned to entities different from the users of those networks. This immediately leads to the need to recover the costs incurred by those entities and to remunerate the investments and assets. This concern was present in transmission networks in the first place due to the emergence of transmission providers, ISO's and Transmission System Operators - TSO's. More recently, the market mechanisms are being extended till the end users so that the discussion on tariffs for use of distribution networks became an issue.

Apart from the regulatory policies that can be adopted for the transmission and distribution wiring companies - see for instance [1, 2, 3] - it still remains an important question regarding the allocation of costs to the users of networks. In the literature one can find a large number of methods to be applied for this purpose but, unfortunately, several of them are not based on sounded grounds, either from physical or economic points of view. These methods can be gathered in three large groups:

\author{
João Tomé Saraiva \\ jsaraiva@inescn.pt \\ Engineering Faculty of University of Porto \\ INESC Porto - Inst. de Engenharia de Sistemas e Computadores \\ Largo de Mompilher, no. 22, 4050-392 Porto, Portugal \\ Phone: +351.2.2..2094230 Fax: +351.2.2.2084172
}

- embedded approaches both using results from power flow studies or not. In this later case, the allocation of costs to users does not even reflect the physical operation of the system so that their transparency and fairness can be immediately questioned. In [4] the author describes an compares a number of these methods;

- incremental approaches comparing costs incurred with and without a given transaction. Methods as the Areas of Influence in use in Chile and Argentina are examples of this type of approaches;

- marginal approaches aiming at evaluating the surplus of cost from increasing load of one unit. They can be divided in short and long term approaches depending on the costs included in the models.

Marginal approaches [5, 6] are well established as the most efficient ones both on their transparency and on the adequacy of the economic signals sent to the users of networks. Unfortunately, short run marginal costs, although easily computed by operation optimization problems as [7, 8, 9, 10], are too volatile thus preventing a stable and predictable stream of money (1). Apart from that, they do not provide the full remuneration of those companies thus leading to revenue reconciliation issues. In expression (1) MR is the remuneration provided via marginal prices, $\rho_{\mathrm{k}}$ is the marginal price at node $\mathrm{k}$ and $d_{k}$ and $g_{k}$ are the load and generation at that node.

$$
\mathrm{MR}=\sum \rho_{\mathrm{k}} \cdot\left(\mathrm{d}_{\mathrm{k}}-\mathrm{g}_{\mathrm{k}}\right)
$$

In fact, marginal prices are influenced by a number of factors as the load level, the generation dispatch policy and component outages that explain large variations of prices in the same node along the time. In order to deal with load uncertainties, the formulation described in [11] reflects load uncertainties represented by fuzzy concepts in the evaluation of nodal marginal prices. Long run marginal costs internalizing investment costs on equipment and operation issues - as congestion and losses - are the most adequate approach to cope with these difficulties. References [12, 13] describe two long term planning approaches leading to the identification of economically adapted transmission system and the most adequate expansion plan and tariff settings considering a number of criteria.

In this paper, long run marginal costs are calculated within a framework aiming at getting economic and technical efficiency from the network by considering operation, investment and reliability related costs as well as technical constraints namely 
related with congestion. This leads to a multiobjective mixed integer problem that is solved by a two step scheme. In the first one, we use a Simulated Annealing approach to generate a set of efficient solutions. This set is then investigated in a more detailed way either by the regulatory agency and/or by the entity in charge of establishing a reference expansion plan for the network. As a result, the methodology provides a reference plan together with the set of nodal prices that lead to a certain level of remuneration collected via marginal concepts. This approach will be illustrated with a case study based on a Portuguese distribution network.

\section{SHORT VERSUS LONG TERM MARGINAL PRICES}

The literature describes a number of models to evaluate short term marginal prices either by:

calculating a price for active power in a significant load node of the system and inducing the spatial dispersion by affecting that price by penalty factors [7];

- considering an explicit representation of the transmission system as in [8,9] and including active transmission losses by successive linearized approaches; adopting an AC model of the power system leading to nodal prices of both active and reactive powers [10];

The spatial and temporally varying marginal prices of electricity - also known as spot prices - are the basis for tariff schemes that aim at allocating operational costs of congestion and losses to the network users. They correspond to the extra cost of electricity due to the increase of 1 unit in the load at a given node $\mathrm{k}$, at an instant $\mathrm{t}$, for a given load level and topology in operation. The expression adopted to compute these prices is model dependent. If we adopt a DC optimization model including a generation/load balance equation and generator, branch and Power Not Supplied limit constraints, expression (2) should be used.

$$
\rho_{\mathrm{k}}=\gamma+\gamma \cdot \frac{\partial \mathrm{L}}{\partial \mathrm{d}_{\mathrm{k}}}-\sum \mu \cdot \frac{\partial \mathrm{P}_{\mathrm{ij}}}{\partial \mathrm{d}_{\mathrm{k}}}+\sigma_{\mathrm{k}}
$$

In this expression $\rho_{\mathrm{k}}$ is the spot price at node $\mathrm{k}$ at instant $\mathrm{t}$ and $\gamma$ is the Lagrange multiplier of the referred load/generation balance equation. $\mathrm{L}$ is the total transmission losses in the system, $P_{i j}$ is power flow in branch $i j$ and $d_{k}$ is the demand at node $\mathrm{k} . \mu$ and $\sigma_{\mathrm{k}}$ are the dual variables of power flow limit constraints and of Power Not Supplied limit constraint in node k. The second term in (2) measures the impact of losses variation deriving from increasing the load of 1 unit in bus $\mathrm{k}$. The third term reflects congestion costs incurred when a branch is at the limit. The fourth term corresponds to the dual variable of the Power Not Supplied constraint in node $\mathrm{k}$ whenever it is on the limit.

This type of marginal pricing is in use, in several countries, as a basis for evaluation of transmission tariffs. Apart from their volatility and their contribution to create a more unstable and unpredictable stream of money in an environment that is already full of uncertainties and risks, short term marginal costs also fail in providing the complete remuneration required by transmission providers. This issue is known as the Revenue Reconciliation problem and, in practice, means that marginal terms in transmission tariffs have to be supplemented by complementary charges, often based on the referred embedded approaches. The reason for the revenue reconciliation problem comes from the fact that short term marginal prices mainly reflect the generation dispatch policy and prices so that the collected remuneration is insufficient and does not reflect the needs of transmission providers. This is particularly true giving that short term or operation models do not include investment costs so that the derived marginal tariffs would not be able to recover them. Although more complex in their computation, long term marginal prices are the most adequate, transparent and fair framework to build tariffs due for the use of networks. As an example, reference [2] details that long term marginal prices should reflect operation, reliability and investment costs regarding a variation $\Delta \mathrm{L}$ of the load (3). In this expression, LTMP is the long term marginal price, $\Delta \mathrm{C}(\mathrm{O}), \Delta \mathrm{C}(\mathrm{R})$ and $\Delta \mathrm{C}(\mathrm{I})$ are the variations of operation, reliability and investment costs due to the load change $\Delta \mathrm{L}$.

$$
\mathrm{LTMP}=\frac{\Delta \mathrm{C}(\mathrm{O})}{\Delta \mathrm{L}}+\frac{\Delta \mathrm{C}(\mathrm{R})}{\Delta \mathrm{L}}+\frac{\Delta \mathrm{C}(\mathrm{I})}{\Delta \mathrm{L}}
$$

The link between long term marginal prices and investment decisions turns the evaluation of these prices more complex and time consuming than short term ones. As examples, reference [12] presents an expansion planning formulation using genetic algorithms as the basic tool to cope with that complexity. In this contribution the authors also discuss the role of planning activities in a market environment and characterize the concept of Economically Adapted System as the basic notion supporting and justifying the identification of reference plans for generation and transmission. In [13] it is described a dynamic programming approach to identify transmission expansion plans and to study the long term effects of transmission tariff settings.

\section{EVALUATION OF LONG TERM MARGINAL PRICES}

\section{A. General Issues}

The contributions referred in Section II are clearly directed to the generation/transmission systems. The recent creation of distribution wiring companies decoupling at this level the circuit of electricity and the flow of money due to the commercialization of electricity imposes a new look at the distribution area. In any case and as referred before, the computation of long term marginal prices is much more complex and involving than the computation of short term costs. This comes from the fact that expansion planning problems:

- $\quad$ are determined by a number of criteria, often having a contradictory nature;

- have a binary nature since we are dealing with investment or expansion on/off decisions;

- $\quad$ are largely affected by uncertainties both in terms of the long term evolution of load values and the availability of system components. 
In order to cope with some of these difficulties we developed a multiobjective decision aid approach directed to the distribution sector in the scope of which we can evaluate long term marginal prices. The approach can be broadly divided in two main steps. In the first one, and after having defined a set of possible expansion plans we identify the set of non-dominated or efficient plans. This is required since we consider a number of criteria as investment, operation and power not supplied costs. This formulation admits loads or independent generation modelled by triangular fuzzy numbers as a way to cope with some of the complexities coming from the uncertain nature of long term problems. Once the efficient solutions are identified, it is conducted a decision analysis in which the Decision Maker can select and analyse more deeply several alternative plans. One of the interesting features of the approach comes from the fact that, on the decision step, we can characterize the available plans by computing the remuneration the distribution wiring company obtains via long term marginal prices. In order to increase the transparency of the tariff setting process, the revenue reconciliation problem should be minimized. This means that, being all the rest equal, a plan providing a larger marginal remuneration should be preferred.

It should be emphasized that this approach should not be classified as an optimization formulation leading to the identification of the best or preferred plan. In fact, the application is more naturally considered a decision aid framework in which we perform a reduction of the list of plans by eliminating the dominated ones and, in second phase, we help the Decision Maker in making a final selection once the remaining plans are fully characterized.

\section{B. Multi-Objective Formulation}

The multi-objective formulation that was adopted corresponds to the model (4) to (11). It supposes that it is available a list of $\mathrm{m}$ expansion plans in terms of building new branches or substations or reinforcing existing ones. As referred before, the input plans are analysed considering three criteria: investment costs (4), operational costs represented by a fuzzy valued function related to active losses (5) and reliability costs also represented by a fuzzy valued function (6).

$$
\begin{aligned}
& \min \mathrm{c}_{\mathrm{I}}=\sum_{\mathrm{i}=1}^{\mathrm{p}} \mathrm{c}_{\mathrm{i}}^{\mathrm{t}} \cdot \delta_{\mathrm{i}} \\
& \min \tilde{\mathrm{c}}_{\mathrm{O}}=\sum_{\mathrm{i}=1}^{\mathrm{p}} \tilde{\mathrm{p}}_{\mathrm{i}}^{\mathrm{t}} \cdot \tilde{\mathrm{x}}_{\mathrm{i}} \\
& \min \tilde{\mathrm{c}}_{\mathrm{R}}=\sum_{\mathrm{i}=1}^{\mathrm{p}} \tilde{\mathrm{e}}_{\mathrm{i}}^{\mathrm{t}} \cdot \tilde{\mathrm{x}}_{\mathrm{i}} \\
& \text { Subj. } \quad \tilde{\mathrm{x}}_{\mathrm{i}}=\mathrm{A}_{\mathrm{i}} \cdot \tilde{\mathrm{d}}_{\mathrm{i}} \\
& \left|\tilde{\mathrm{x}}_{\mathrm{ki}}\right| \leq \gamma_{\mathrm{ki}} \cdot \overline{\mathrm{x}}_{\mathrm{k}} \\
& \mathrm{i}=1 \ldots \mathrm{p} \\
& \left|\Delta \tilde{U}_{j i}\right| \leq \Delta U_{\text {max }} \quad i=1 . . p, j=1 . . m \\
& \sum_{\mathrm{i}=1}^{\mathrm{p}} \delta_{\mathrm{ki}} \leq 1 \quad \mathrm{k}=1 . . \mathrm{m} \\
& \gamma_{\mathrm{ki}} \geq \sum_{\mathrm{j} \leq \mathrm{i}} \delta_{\mathrm{kj}} \quad \mathrm{k}=1 . . \mathrm{m}
\end{aligned}
$$

The model outputs the most adequate decisions about building new facilities $\left(\delta_{\mathrm{ki}}\right)$ during the p periods in which the planning horizon was divided. The fuzzy nature of criteria (5) and (6) reflect the triangular fuzzy numbers adopted to model load uncertainties. For this fuzzy load scenario, we perform an AC Fuzzy Power Flow study whose basic ideas were presented in [14]. This study leads to fuzzy power flows, currents and voltage drops and, whenever that is required, to a fuzzy description of power not supplied. The formulation also includes constraints on maximum branch flows $\tilde{\mathrm{x}}_{\mathrm{ij}}(8)$ and on maximum voltage drop $\Delta \tilde{\mathrm{U}}_{\mathrm{ij}}(9)$ as well as constraints to ensure that new configurations keep their radial nature (not represented in the above formulation). Finally, some more variables and parameters are included in the model:

$\mathbf{c}_{\mathbf{i}}, \tilde{\mathbf{p}}_{\mathbf{i}}, \tilde{\mathbf{e}}_{\mathbf{i}}$ are the costs of building new branches, fuzzy cost per unit of losses and fuzzy cost per unit of power not supplied;

$\gamma_{\mathrm{ki}}$ represents the existence or not of facilities included in plan $\mathrm{k}$ in period $\mathrm{i}$;

- $\delta_{\mathrm{ki}}$ indicates the construction or not of the facilities included in plan $\mathrm{k}$ in period $\mathrm{i}$;

It should be referred that the fuzzy characterization of loads leads to a more flexible, robust and adapted approach. In fact, in several planning situations, the planner has not enough information about the past behavior of relevant parameters or the laws determining their evolution may have changed. In these cases, the adoption of probabilistic techniques is conceptually inappropriate. Nevertheless, subjective knowledge available from planners and based on their past experience is relevant and most valuable in the planning environment. Fuzzy Sets emerged in the $60^{\text {th }}$ exactly as a powerful mathematical and rigorous tool to model this knowledge, to translate it into mathematical entities and to integrated it into several models. The adoption of fuzzy set concepts implies that, in several situations, rules from fuzzy arithmetic are used instead of traditional ones. As a final result, this formulation provides the evaluation and characterization of alternative investment plans, also considering their impact on the technical operation of the network, provided those plans are included in the initially specified list.

\section{Solution Approach}

As indicated in Section III.A the solution approach comprises two main steps corresponding to the identification of the set of efficient plans and to a Decision phase. Regarding the identification of efficient plans the literature indicates two traditional approaches: the weighting approach and the $\varepsilon$ constrained method. The first one aggregates all objectives in a single function considering weights. If these weights are strictly positive, reference [15] proves that the solution of the new formulation is a non-dominated solution of the original problem. By changing these weights it is therefore possible to identify a set of non-dominated solutions that, however, are delimited by a convex envelope. In the second approach this limitation is not present thus leading to a framework more adapted to real discrete problems. The $\varepsilon$-constrained method consists of 
transforming all criteria but one in constraints for which we specify bounds. In this case, the variation of these specified bounds leads to the identification of non-dominated solutions.

In our approach and for the above detailed reason, we adopted the $\varepsilon$-constrained method. However, this does not solve the problem by itself since the discrete nature of the investment problem requires a powerful optimization technique. Therefore, we selected Simulated Annealing [16] as the basic optimization technique for its ability to treat discrete problems in a very natural and efficient way. In a $\mathrm{n}$ criteria problem and once a criterium is selected and the remaining $n-1$ ones originate constraints in terms of the specified bounds, we are restricting the search to an hypercube in a n-1 dimensional space. As an example, in our formulation we originally have three criteria. This means that, selecting the investment cost and imposing bounds to operational and reliability costs we are imposing that the algorithm searches for the optimal solution in a square delimited in the attribute space by the bounds imposed on operation and reliability costs. Changing these bounds directly determines changing the square where the search is being conducted.

\section{Computation of Long Term Marginal Prices}

Once a non dominated plan is identified we compute the corresponding nodal long term marginal prices. This evaluation is performed considering that the variation of the value of the objective function corresponds, for each nodal load increment, to that node long term marginal price. This approach leads to a large computational time that should be interpreted as the price to pay for having adopted a discrete formulation for the investment problem. It should be referred that this is not crucial since we are at a planning environment. This is why we decided to adopt a more accurate representation of the investment problem - leading to a discrete formulation - given the implications it has on prices and on the required remuneration of network providers.

\section{E. Extra Indices to Characterize Non Dominated Plans}

Still before entering in the decision step we evaluate two extra indices in order to characterize more completely each nondominated plan by two extra indices.

The first one corresponds to the remuneration provided by the corresponding set of long term marginal prices. As referred in Section II the revenue reconciliation problem should be minimized and this can be accomplished by preferring plans for which the marginal remuneration is larger, being the rest similar. As this marginal remuneration increases and approaches the global remuneration of the distribution wiring company, the complementary charge is more and more reduced. This reduces the margin of subjectivity and lack of transparency and accountability given that the complementary charge is often obtained by the application of poorly sounded embedded methods.

The second index corresponds to a risk index measuring the ability the system has to accommodate the specified load uncertainties without leading to voltage drops or thermal branch limit violations. This risk index computed in terms of a Robustness Index is evaluated as follows. For each fuzzy membership function built for voltage drops and branch currents we compute Partial Robustness Indices $-\mathrm{PRI}_{\mathrm{i}}$ - as illustrated in Figure 1. In this Figure one represents a triangular fuzzy number corresponding, for instance, to the current flow in branch i. We consider three situations regarding the relative location of this fuzzy representation and the maximum current flow $\mathrm{I}^{\mathrm{max}}$. In Case I, the system is completely unable to accommodate the specified uncertainties without violating the flow limit. Therefore, situation (12) holds. In Case II, it may occur a violation of $I^{\max }$ at level $\alpha$. This leads to the PRI $\mathrm{i}_{\mathrm{i}}$ value indicated in (13). Finally, in Case III, no matter the load values considered, $\mathrm{I}^{\max }$ is never violated. In this case the system is fully robust when analysing the flow in this branch so that we have (14). For a complete network, having a set of partial $\mathrm{PRI}_{\mathrm{i}}$ related to voltage drops and branch flows, the global RI value is given by (15).

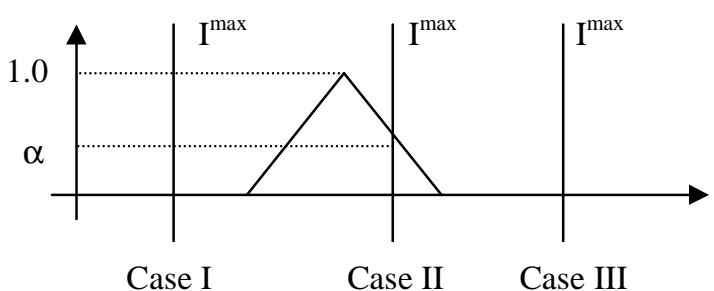

Fig. 1 - Illustration of the computation of $\mathrm{PRI}_{\mathrm{i}}$.

$$
\begin{aligned}
& \text { Case } \mathrm{I} \rightarrow \mathrm{PRI}_{\mathrm{i}}=0.0 \\
& \text { Case } \mathrm{II} \rightarrow \mathrm{PRI}_{\mathrm{i}}=1.0-\alpha \\
& \text { Case } \mathrm{III} \rightarrow \mathrm{PRI}_{\mathrm{i}}=1.0 \\
& \mathrm{RI}=\min \left(\mathrm{PRI}_{\mathrm{i}}\right)
\end{aligned}
$$

The remuneration obtained via marginal prices and the global Robustness Index are used to provide the Decision Maker a more complete characterization of the remaining plans.

\section{F. Decision Making Step}

In the whole, the three referred criteria together with the marginal remuneration and the Robustness Index characterize each expansion plan and their values are presented to the Decision Maker to perform the final decision process. This gives the Decision Maker a very important amount of information characterizing each efficient plan so that the decision process can be conducted in a more sounded and robust way. However, if the number of available efficient plans is large, the final decision process will be complex. In these cases, the activity of the Decision Maker should be supported by specific decision analysis tools that are discussed, for instance, in [17].

\section{CASE STUDY}

In this section we illustrate the application of the developed long term distribution planning approach to a case study based on a Portuguese MV distribution network. This network belongs 
to a Center-West region of Portugal and was kindly made available by a Portuguese Utility. It includes 51 nodes, 75 overhead lines and cables and it is supplied by two HV/MV substations.

In the planning model we used load forecasts provided by the utility for three future periods. In order to cope with uncertainties affecting load values we considered that those nodal forecasts correspond to the Central Value of the triangular fuzzy number that is adopted to represent the load in each node. The Central Value of a fuzzy number just corresponds to the mean value of all loads to which it was assigned the degree of membership 1.0. In the particular case of triangular fuzzy numbers the Central Value just corresponds to the unique value having membership degree 1.0. The range of load values of each triangular fuzzy number at the 0.0 level of uncertainty increases when passing from period 1 to period 2 and from this one to period 3 . These ranges are typically $\pm 10 \%$ for period $1, \pm 15 \%$ for period 2 and $\pm 20 \%$ for period 3 . These larger uncertainty ranges were adopted in order to reflect the reduction on the credibility of forecasts as the planning horizon moves deeper and deeper into the future.

Data for several parameters, namely reliability data, were specified considering typical values available in the literature. Several additional details regarding this network can be obtained from the authors and are published in [17].

The list of possible expansion plans includes 240 alternatives. These alternatives were generated considering several hypothesis of possible reinforcement of one of the two substations of the network, the reinforcement of several sets of branches and the installation of new lines and cables thus leading to different topologies. In any case, it was assumed that all those new topologies remained radial since this was a common operational practice at the referred utility for that voltage level.

In the first place, we performed the multiobjective optimization process corresponding to the first phase referred in Sections III.B and III.C. This process lead to the identification of the set of efficient solutions corresponding to expansion plans or to the reinforcement of existing installations. As an example, Table I presents the values of the attributes of the problem for 6 efficient solutions identified in this process. In particular, Table I includes that values for Investment Costs, Power Not Supplied, Robustness Index and Losses. The last one is adopted as a way to measure, at least partially, operational costs. As referred before, the Robustness Index aggregates information regarding the quality of the solution in what concerns the violation of limits imposed on voltage drops and on branch current flows.

Once the efficient alternatives are identified, the nodal marginal prices were computed by running a set of optimization studies increasing each load at time by one unit. In Figures 2 and 3 we present graphically the marginal prices obtained for two alternative plans. The graph in Figure 4 presents the average nodal marginal prices for the set of efficient solutions.
TABLE I - Attributes for 6 efficient solutions.

\begin{tabular}{cccccc}
\hline Solution & Cost $\left(10^{6} \$\right)$ & Robustness & Losses $(\mathrm{kW})$ & PNS $(\mathrm{MW})$ & Revenue $\left(10^{6} \$\right)$ \\
\hline 75 & 242.98 & 0.57 & 543.00 & 3.5 & 20.43 \\
89 & 209.64 & 0.57 & 612.00 & 3.5 & 23.75 \\
190 & 303.19 & 1.0 & 424.43 & 2.67633 & 21.51 \\
194 & 298.01 & 1.0 & 444.41 & 3.10114 & 22.98 \\
79 & 320.76 & 1.0 & 437.76 & 2.70196 & 23.65 \\
42 & 247.76 & 1.0 & 642.44 & 3.52596 & 19.95 \\
\hline
\end{tabular}

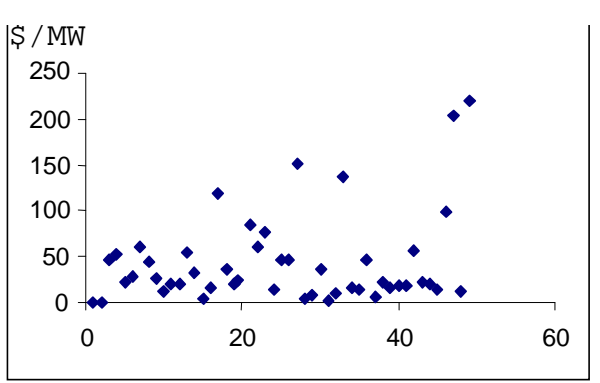

Fig. 2 - Marginal prices obtained for plan 42 (\$/MW).

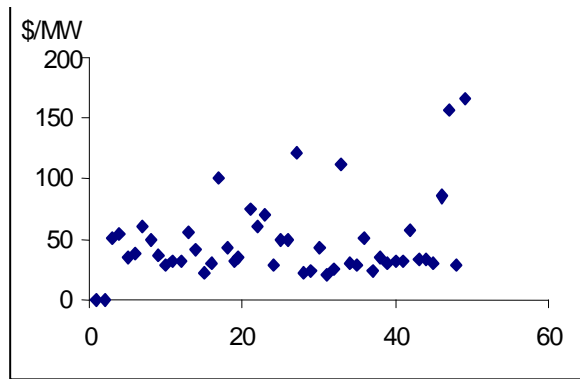

Fig. 3 - Marginal prices obtained for plan 75 (\$/MW).

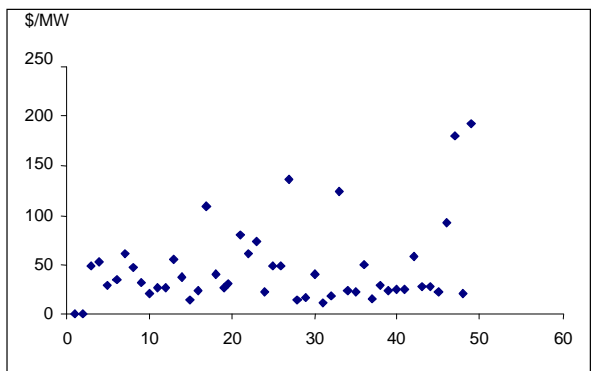

Fig. 4 - Average values of Marginal Prices (\$/MW).

When analysing the values in Figures 2 and 3 one can see that marginal prices display a large geographical variability explained by the investment decisions and by the different operational costs that are included in the model. However, in these 2 cases, there is a common global pattern followed by the marginal prices. This is also confirmed by the average values of the nodal prices obtained for all efficient solutions and depicted in Figure 4.

As a final remark for the related remunerations we point out that according to expression (1), once these marginal prices are computed, one can evaluate the remuneration obtained by the distribution provider via the long term marginal based tariffs. The remuneration obtained this way is indicated in the right column of Table I for each solution and assuming a flat load diagram. Surely, this is an approximate value for the marginal 
remuneration since, in a real world planning problem, it should be used a more detailed temporarily varying load forecast.

These tariffs would provide a base remuneration immune to volatility issues and its value could also be used by the decision maker in anticipating how an expansion investment would impact on the revenue reconciliation problem. Since we are not dealing with short term load variations (for instance, from daily peak to valley hours) this remuneration would certainly not cover the entire costs of the distribution provider. In any case, from the point of view of the tariff debate and being all criteria detailed in Section III comparable, it would certainly be preferred an expansion plan that would allow the distribution provider to obtain a remuneration covered as largely as possible by these stable marginal prices. In this sense, the remuneration provided by long term marginal prices would also be integrated in the decision process as a way to characterize in a more complete way each efficient solution.

\section{CONCLUSIONS}

In this paper we presented a novel approach to compute long term marginal prices in the scope of a long term distribution planning process. The formulation integrates a number of criteria that are relevant in the planning process and is able to integrate load uncertainties represented by fuzzy concepts. After identifying the efficient solutions, the Decision Maker has more complete information in order to conduct a Decision Process. At this point he may need further support because the list of efficient solutions usually integrates a large number of alternatives. Nevertheless, the literature presents several methods aiming at reducing the large number of alternatives into a reduced set. This reduction process should be conducted in a careful and systematic way so that the reduced list is still representative of the complete set of efficient solutions. This reduction has the advantage of simplifying the Decision Process while maintaining the confidence of the Decision Maker in the process. This way, this research will be completed in the future by integrating a Decision Aid Tool in order to help the Decision Maker in taking his final decision.

Finally, it should be stressed that this kind of methodologies enables Regulatory Boards or network providers to anticipate the consequences of adopting a regulatory policy or set new tariffs levels since the remuneration obtained via marginal prices can be computed. This may contribute to eliminate one of the factors causing uncertainty and volatility in today's electric industry.

\section{ACKNOWLEDGMENT}

This work was partially financed by contract PRAXIS 2/2.1/TIT/1634/95

\section{REFERENCES}

[1] T. Gomez, "Incentive Regulation for Distribution Companies Under Electricity Competition”, available http://www.iit.upco.es;

[2] E. Kahn, Electric Utility Planning \& Regulation, American Council for an Energy-Efficient Economy, Washington DC, USA, 1988.

[3] L. Philipson, H. L. Willis, Understanding Electric Utilities and
DE-Regulation, Marcel Dekker, USA, 1999.

[4] J. Maragon Lima, "Allocation of Transmission Fixed Charges: An Overview", IEEE Trans. Power Systems, vol. 11, no. 3, August 1996.

[5] M. C. Caramanis, R. E. Bohn, F. C. Schweppe, "Optimal Spot Pricing: Practice and Theory", IEEE Trans. Power Systems, vol. 101, no. 9, September 1982.

[6] F. Schweppe, M. Caramanis, R. Tabors, R. Bohn, Spot Pricing of Electricity, Kluwer Academic Publishers, London, 1988.

[7] R. Palma, H. Rudnick, H. Lira, "Penalty Factor Calculations for Marginal Pricing of Transmission Systems in a Hydroelectrical System", Proceedings of Stockholm Power Tech, SPT'95, June 1995.

[8] M. Rivier, I. Pérez-Arriaga, "Computation And Decomposition of Spot Prices for Transmission Pricing", $11^{\text {th }}$ Power Systems Computation Conference, PSCC'93, Avignon, 1993.

[9] F. J. Rubio, I. Pérez-Arriaga, "Marginal Pricing of Transmission Services: A Comparative Analysis of Network Cost Allocation Methods", in Power Engineering Review, November 1997.

[10] A. El-Keib, X. Ma, "Calculating Short-Run Marginal Costs of Active and Reactive Power Production", IEEE Trans. Power Systems, vol. 12, no. 2, May 1997.

[11] J. T. Saraiva, "Evaluation of the Impact of Load Uncertainties in Spot Prices Using Fuzzy Set Models", $13^{\text {th }}$ Power Systems Computation Conference, PSCC'99, Trondheim, July 1999.

[12] H. Rudnick, R. Palma, E. Cura, C. Silva, "Economically Adapted Transmission Systems in Open Access Schemes - Application of Genetic Algorithms", IEEE Trans. Power Systems, vol. 11, no. 3, August, 1996.

[13] J. Maragon Lima, E. Oliveira, "The Long Term Impact of Transmission Pricing”, IEEE Trans. Power Systems, vol. 13, no. 4, November 1998.

[14] V. Miranda, M. A. Matos, J. T. Saraiva, "Fuzzy Load Flow- New Algorithms Incorporating Uncertain Generation and Load Representation", Proceedings of the 10th Power Systems Computation Conference, Butterworths, London, August 1990.

[15] A. Goicoechea, D. R. Hansen, L. Duckstein, 1982, Multiobjective Decision Analysis with Engineering Business Applications, John Wiley \& Sons, New York.

[16] E. Aarts, J. Korst, Simulated Annealing and Boltzman Machines, John Wiley \& Sons, New York, 1990.

[17] M. T. Ponce de Leão, M. A. Matos, "Multicriteria Distribution Network Planning Using Simulated Annealing", International Transactions in Operational Research, Vol.6/4, pp 337-391, Elsevier Science, 1999.

Maria Teresa Ponce de Leão was born in Porto, Portugal, on August 13, 1957. She received his licenciate, M.S. equivalent and Ph.D. degrees from the Faculty of Engineering at Porto University (FEUP) in 1980, 1987 and 1996, respectively, in Electrical and Computers Engineering. Currently she is a Professor in the Electrical and Computers Department of FEUP. In 1987 she joined also INESC as a researcher. In recent years, she has been involved in the development of DMS systems and in projects related with the evaluation of the impact of independent generation in distribution planning.

João Tomé Saraiva was born in Porto, Portugal in 1962. In 1985 and 1993 he got his degree and $\mathrm{PhD}$ in Electrical and Computer Engineering from Faculdade de Engenharia da Universidade do Porto (FEUP). In 1985 he joined INESC Porto - a private research institute where he is research manager. Currently he is also Professor in the Electrical and Computers Department of FEUP. He was head researcher or collaborated in several projects related with the development of DMS systems, quality in power systems in the scope of re-regulation and tariffs due for the use of transmission and distribution networks. 